\title{
Revenus des médecins indépendants de Suisse en 2001 et 2002
}

\section{Niklaus Hasler}

Rapport d'analyse établi sur mandat de la Fédération des médecins suisses (FMH). Le rapport complet, constitué de 59 pages, 27 tableaux et 28 graphiques, se trouve sur le site internet de la FMH www.fmh.ch $\rightarrow$ Deutsch $\rightarrow$ Über uns $\rightarrow$ Ärzteeinkommen. La numérotation des tableaux et graphiques se rapporte à ce rapport.

Correspondance: Dr Niklaus Hasler Huzlenstrasse 3 8604 Volketswil nik@hin.ch

\section{A. Introduction}

Le 28 janvier 2005, la Fédération des médecins suisses a donné mandat d'analyser les revenus des médecins indépendants de Suisse pour 2002 à nouveau au moyen d'une saisie complète.

\section{A.1.1. Remarque concernant TARMED}

Le nouveau tarif médical valable dans toute la Suisse a été introduit le $1^{\text {er }}$ mai 2003 pour les traitements relevant de la loi sur l'assuranceaccidents, de l'assurance-invalidité et de la loi sur l'assurance-militaire (volume d'honoraires estimé pour 2003 à 5,3\% du volume total brut des honoraires) puis le $1^{\text {er }}$ janvier 2004 pour tous les traitements selon la loi sur l'assurance-maladie (de sorte que le total du volume brut des honoraires est facturé selon le TARMED).

La présente analyse des revenus 2002 des médecins est ainsi la dernière qui soit effectuée entièrement sans l'application du TARMED.

La prochaine analyse concernera les revenus 2003 (elle paraîtra en automne 2006); elle comprendra déjà une partie des traitements facturés selon le TARMED (env. 5,3\% du volume brut des honoraires). Le rapport suivant concernera les revenus 2004 (il paraîtra en automne 2007) et ne comprendra plus que des revenus bruts facturés selon le TARMED.

La présente analyse fait directement suite aux 20 premiers rapports établis par le Dr Niklaus Hasler entre le mois d'août 1977 et le mois de juillet 2004.

Vous trouverez dans les lignes qui suivent un résumé des résultats les plus importants ainsi que des tableaux et des graphiques. Ce résumé ne contient sciemment que les chiffres pour les médecins âgés de moins de 66 ans. La version complète contient toutefois aussi les chiffres pour les médecins âgés de plus de 65 ans et ceux pour tous les médecins (indépendamment de l'âge).

En outre, d'autres critères d'analyse sont appliqués dans la version complète, comme le rapport entre le revenu, d'une part, et la masse salariale du personnel, l'âge du médecin ou le nombre d'années en pratique indépendante depuis l'ouverture du cabinet d'autre part.
Le chapitre suivant présente le changement le plus important intervenu dans la saisie pour la période 1997/98 à 2001, à savoir le passage de l'imposition sur la base du revenu obtenu pendant une période antérieure (revenu antérieur) à l'imposition sur la base du revenu de l'année concordant à l'année fiscale (revenu actuel).

\section{A.2. Passage à l'imposition sur la base du revenu actuel}

La taxation des cotisations AVS des indépendants a également passé de l'imposition du revenu antérieur à celle du revenu actuel lors de l'harmonisation fiscale au niveau fédéral.

Le revenu moyen des années 1997 et 1998 (30 période d'impôt de défense nationale) constitue la base décisionnelle des cotisations AVS personnelles à payer pour 1999 et 2000.

Les revenus professionnels 1999 et 2000 tombent dans la période sans évaluation et ne peuvent donc pas être saisis ni analysés.

A partir de 2001, c'est l'imposition sur le revenu actuel qui est applicable, c.-à-d. que l'année de revenu 2001 correspond aussi à l'année de cotisations AVS 2001.

Jusqu'aux revenus de la $30^{\text {e }}$ période d'impôt de défense nationale (années fiscales 1997/98), les revenus des médecins ne pouvaient être saisis qu'en tant que valeur moyenne calculée sur deux ans à intervalles réguliers de deux ans.

A partir de 2001, les revenus des médecins sont saisis chaque année en tant que revenus annuels sur la base de la taxation des cotisations AVS personnelles.

\section{A.3. Problèmes liés au passage}

La structure fédéraliste de notre pays fait que les lois ne sont pas appliquées au même moment dans tous les cantons. Pour cette raison, l'analyse de juillet 2004 a eu quelques difficultés avec les cantons du Tessin, du Valais et de Vaud.

Aujourd'hui, on constate avec toute la clarté voulue que l'ensemble des cantons a passé à l'imposition sur le revenu actuel pour évaluer les cotisations AVS personnelles. 


\section{Résultats}

\section{D.1. Généralités}

Aux fins de vérification, les chiffres 2001 publiés en juillet 2004 ont été repris et analysés une nouvelle fois dans le rapport actuel. Les valeurs révisées qui sont maintenant publiées sont légèrement inférieures mais malgré tout très proches des valeurs de la saisie de juillet 2004:

- Pour tous les médecins, indépendamment de l'âge: (valeur moyenne du revenu assujetti à l'AVS: 203855 au lieu de $205466=-0,78 \%$; valeur centrale du revenu assujetti à l'AVS: 164700 au lieu de $165200=-0,30 \%$ ).

- Pour tous les médecins âgés de moins de 66 ans: (valeur moyenne du revenu assujetti à l'AVS: 210723 au lieu de $212494=-0,83 \%$; valeur centrale du revenu assujetti à l'AVS: 171500 au lieu de $172200=-0,41 \%$ ).

- Pour tous les médecins âgés de plus de 65 ans: (valeur moyenne du revenu assujetti à l'AVS: 92081 au lieu de $94152=-2,20 \%$; valeur centrale du revenu assujetti à l'AVS: 45100 au lieu de $40800=+10,54 \%$ ).

Les différences ne sont de loin pas les mêmes par spécialité. Voici quelques exemples:

- Pour les gynécologues âgés de moins de 66 ans (valeur moyenne du revenu assujetti à l'AVS: 249477 au lieu de $253434=-1,56 \%$, valeur centrale du revenu assujetti à l'AVS: 219100 au lieu de $220500=-0,63 \%$ ).

- Pour les urologues âgés de moins de 66 ans (valeur moyenne du revenu assujetti à l'AVS: 312410 au lieu de $329133=-5,08 \%$, valeur centrale du revenu assujetti à l'AVS: 256900 au lieu de $270800=-5,13 \%$ ).

- Pour les psychiatres âgés de moins de 66 ans (valeur moyenne du revenu assujetti à l'AVS: 117513 au lieu de $117389=+0,11 \%$, valeur centrale du revenu assujetti à l'AVS: 107500 au lieu de $110100=-2,36 \%$ ).

- Pour les praticiens généralistes âgés de moins de 66 ans (valeur moyenne du revenu assujetti à l'AVS: 194738 au lieu de $198139=$ $-1,72 \%$, valeur centrale du revenu assujetti à l'AVS: 178000 au lieu de $181600=-1,98 \%$ ).

A l'instar des valeurs absolues pour les revenus 2001, les revenus 1997/98 et 2001 sont corrigés de la façon suivante.

Evolution des revenus 1997/98 à 2001

Comparaison entre l'analyse de juillet 2004 et celle d'octobre 2005.
Renchérissement 1997/98 à 2001: +3,40\%.

Modification du revenu moyen assujetti à l'AVS 1997/98 à 2001:

- tous les médecins, indépendamment de l'âge: $+2,53 \%$ au lieu de $+3,37 \%$;

- médecins âgés de moins de 66 ans: +2,98\% au lieu de $+3,85 \%$;

- médecins âgés de plus de 65 ans: +7,88\% au lieu de $+10,30 \%$.

Modification du revenu central assujetti à l'AVS 1997/98 à 2001:

- tous les médecins, indépendamment de l'âge: $+0,67 \%$ au lieu de $+0,98 \%$;

- médecins âgés de moins de 66 ans: $+1,18 \%$ au lieu de $+1,59 \%$;

- médecins âgés de plus de 65 ans: -13,77\% au lieu de $-21,99 \%$.

\section{Evolution des revenus 2001 à 2002}

La hausse du renchérissement entre 1997/98 et 2001 s'est élevée à $+0,63 \%$. Durant la même période, le revenu moyen (revenu professionnel assujetti à l'AVS) a évolué comme suit en Suisse pour les groupes de médecins indépendants suivants:

- tous les médecins: de 203805 à 207 752; augmentation de $+1,94 \%$;

- médecins âgés de moins de 66 ans: de 210723 à 215071 ; augmentation de $+2,06 \%$;

- médecins âgés de plus de 65 ans: de 92081 à 91889; diminution de $-0,21 \%$.

Le revenu central des médecins (la moitié des médecins ont un revenu inférieur au revenu central et l'autre moitié un revenu supérieur) a augmenté durant la même période pour les mêmes groupes de la manière suivante:

- tous les médecins: de 164700 à 167 900; augmentation de $+1,94 \%$;

- médecins âgés de moins de 66 ans: de 171500 à 173800 ; augmentation de $+1,34 \%$;

- médecins âgés de plus de 65 ans: de 45100 à 48000 ; augmentation de $+6,43 \%$.

A l'exception des médecins âgés de plus de 65 ans, le revenu moyen a, en 2001 et 2002, augmenté à peine plus que le renchérissement. On constate une augmentation réelle entre 1,31\% et 1,43\%. Pour le revenu central en revanche, on enregistre une augmentation réelle entre $0,71 \%$ et $1,31 \%$.

Pour les médecins âgés de plus de 65 ans, on constate une diminution réelle de $-0,84 \%$ pour le revenu moyen et une augmentation réelle de $+5,80 \%$ pour le revenu central. 


\section{Tableau 23a}

Indices du revenu assujetti à l'AVS des médecins indépendants de Suisse (jusqu'en 1975/76 âgés de moins de 63 ans, dès $1977 / 78$ de moins de 66 ans). 1971/72 à 2002: première partie 1971/72 à 1983/84. Toutes les lignes d'indice sont calculées sur la base 1971/72 = 100.0.

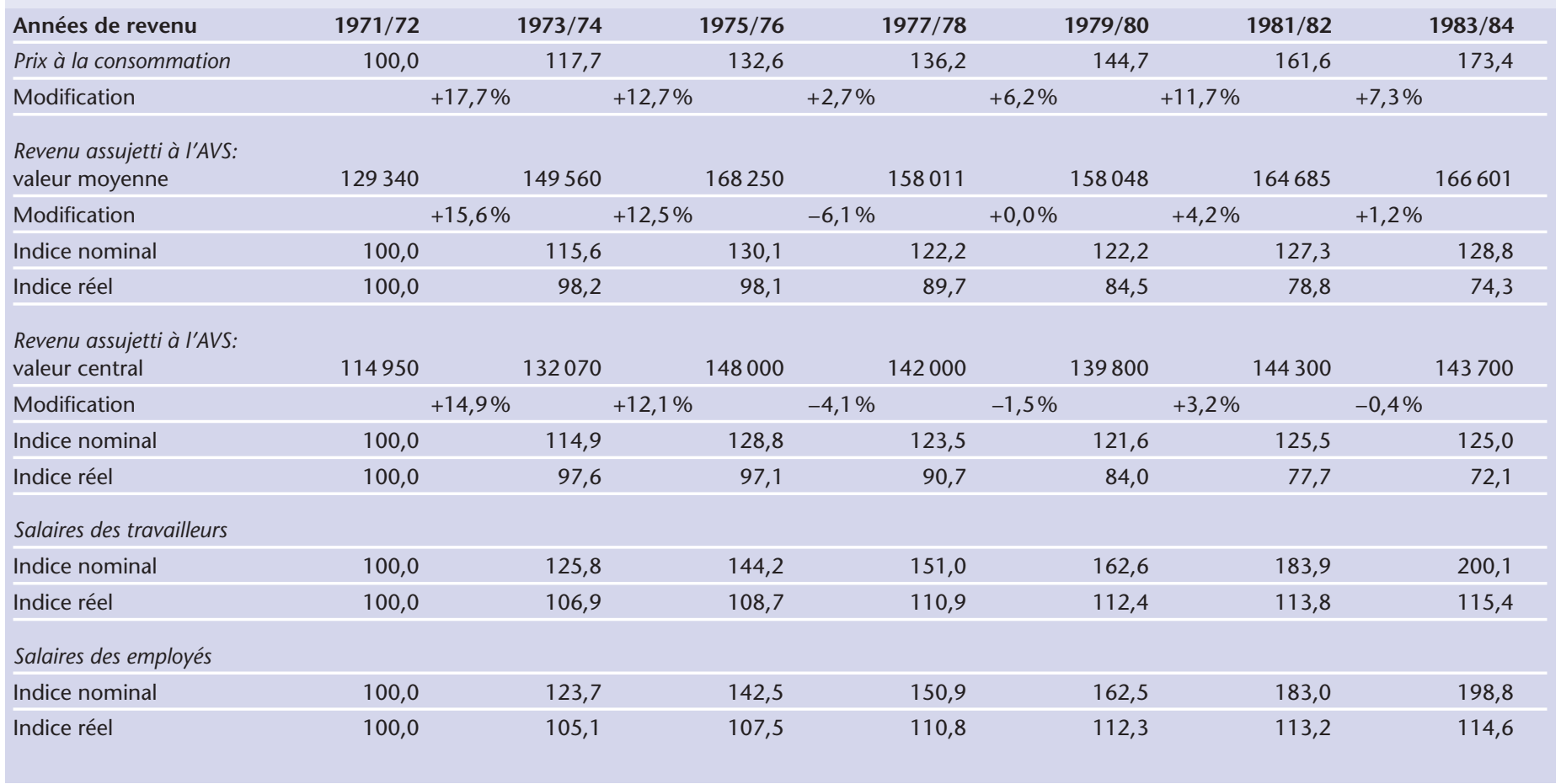

\section{Tableau $23 \mathrm{~b}$}

Indices du revenu assujetti à l'AVS des médecins indépendants de Suisse (jusqu'à 1975/76 âgés de moins de 63 ans, dès $1977 / 78$ de moins de 66 ans). 1971/72 à 2002: deuxième partie 1983/84 à 1995/96. Toutes les lignes d'indice sont calculées sur la base des années $1971 / 72$ = 100.0 .

\begin{tabular}{|c|c|c|c|c|c|c|c|c|c|}
\hline Années de revenu & $1983 / 84$ & & $1985 / 86$ & & $1987 / 88$ & $1989 / 90$ & $1991 / 92$ & $1993 / 94$ & $1995 / 96$ \\
\hline Prix à la consommation & 173,4 & & 182,6 & & 187,7 & 200,7 & 222,4 & 235,3 & 241,6 \\
\hline Modification & & $+5,3 \%$ & & $+2,8 \%$ & & $+6,9 \%$ & $+10,8 \%$ & $+5,8 \%$ & $+2,7 \%$ \\
\hline $\begin{array}{l}\text { Revenu assujetti à I'AVS: } \\
\text { valeur moyenne }\end{array}$ & 166601 & & 168423 & & 184599 & 189044 & 201577 & 209092 & 209529 \\
\hline Modification & & $+1,1 \%$ & & $+9,6 \%$ & & $+2,4 \%$ & $+6,6 \%$ & $+3,7 \%$ & $+0,2 \%$ \\
\hline Indice nominal & 128,8 & & 130,2 & & 142,7 & 146,2 & 155,9 & 161,7 & 162,0 \\
\hline Indice réel & 74,3 & & 71,3 & & 76,0 & 72,8 & 70,1 & 68,7 & 67,1 \\
\hline $\begin{array}{l}\text { Revenu assujetti à I'AVS: } \\
\text { valeur central }\end{array}$ & 143700 & & 143600 & & 154500 & 155500 & 163900 & 170700 & 171500 \\
\hline Modification & & $-0,4 \%$ & & $+7,6 \%$ & & $+0,6 \%$ & $+5,4 \%$ & $+4,1 \%$ & $+0,5 \%$ \\
\hline Indice nominal & 125,0 & & 124,9 & & 134,4 & 135,3 & 142,6 & 148,5 & 149,2 \\
\hline Indice réel & 72,1 & & 68,4 & & 71,6 & 67,4 & 64,1 & 63,1 & 61,8 \\
\hline \multicolumn{10}{|l|}{ Salaires des travailleurs } \\
\hline Indice nominal & 200,1 & & 212,8 & & 226,0 & 246,6 & 279,8 & 296,3 & 303,7 \\
\hline Indice réel & 115,4 & & 116,5 & & 120,4 & 122,9 & 125,8 & 125,9 & 125,7 \\
\hline \multicolumn{10}{|l|}{ Salaires des employés } \\
\hline Indice nominal & 198,8 & & 211,8 & & 223,9 & 242,0 & 269,8 & 285,1 & 293,1 \\
\hline Indice réel & 114,6 & & 116,0 & & 119,3 & 120,6 & 121,3 & 121,1 & 121,3 \\
\hline
\end{tabular}


Tableau 23c

Indices du revenu assujetti à I'AVS des médecins indépendants de Suisse (jusqu'à 1975/76 âgés de moins de 63 ans dès 1977/78 de moins de 66 ans). 1971/72 à 2002: troisième partie 1995/96 à 2002. Toutes les lignes d'indice sont calculées sur la base des années 1971/72 = 100.0.

\begin{tabular}{|c|c|c|c|c|c|c|}
\hline Années de revenu & $1995 / 96$ & & $1997 / 98$ & & 2001 & 2002 \\
\hline Prix à la consommation & 241,6 & & 243,8 & & 252,1 & 253,7 \\
\hline Modification & & $+0,9 \%$ & & $+3,4 \%$ & & \\
\hline $\begin{array}{l}\text { Revenu assujetti à I'AVS: } \\
\text { valeur moyenne }\end{array}$ & 209529 & & 204624 & & 210723 & 215071 \\
\hline Modification & & $-2,3 \%$ & & $+3,0 \%$ & & \\
\hline Indice nominal & 162,0 & & 158,2 & & 162,9 & 166,3 \\
\hline Indice réel & 67,1 & & 64,9 & & 64,6 & 65,5 \\
\hline $\begin{array}{l}\text { Revenu assujetti à l'AVS: } \\
\text { valeur centrale }\end{array}$ & 171500 & & 169500 & & 171500 & 173800 \\
\hline Modification & & $-1,2 \%$ & & $+1,2 \%$ & & \\
\hline Indice nominal & 149,2 & & 147,5 & & 149,2 & 151,2 \\
\hline Indice réel & 61,8 & & 60,5 & & 59,2 & 59,6 \\
\hline \multicolumn{7}{|l|}{ Salaires des ouvriers } \\
\hline Indice nominal & 303,7 & & 307,3 & & 319,8 & 325,77 \\
\hline Indice réel & 125,7 & & 126,1 & & 126,9 & 128,4 \\
\hline \multicolumn{7}{|l|}{ Salaires des employés } \\
\hline Indice nominal & 293,1 & & 298,2 & & 312,6 & 317,7 \\
\hline Indice réel & 121,3 & & 122,3 & & 124,0 & 125,2 \\
\hline
\end{tabular}

Si l'on peut une nouvelle fois analyser ces chiffres dans une ou deux années avec les taxations AVS définitives et qu'il en ressort des valeurs inférieures de 2 à 3 pour cent, l'évolution hors renchérissement et la petite augmentation réelle se transformeront à coup sûr en une perte réelle de revenu.

\section{Evolution des revenus de 1971/72 à 2002}

Avec les chiffres révisés 2001 et les nouveaux chiffres 2002, il est maintenant possible de suivre l'évolution du revenu moyen et central assujetti à l'AVS des médecins indépendants de Suisse sur trois décennies.

Les graphiques 13 à 16 (annexe pages 52/53) présentent les lignes d'indice pour le revenu moyen et le revenu central assujettis à l'AVS (uniquement médecins âgés de moins de 66 ans), aussi bien nominal que réel (c.-à-d. avec prise en considération du renchérissement).

Parmi les nombreux tableaux et graphiques du rapport original (enregistré sur le site internet de la FMH www.fmh.ch), nous prenons ici comme exemples les graphiques 13 et 14 (évolution de la valeur moyenne du revenu professionnel assujetti à l'AVS des médecins indépendants de Suisse âgés de moins de 66 ans, nominal et réel, avec prise en compte du renchérissement) et les tableaux 5 et 9 (moyenne du revenu assujetti à l'AVS 2001 et 2002 des médecins indépendants âgés de moins de 66 ans d'après les cantons et les spécialités).

\section{F.8. Dépenses globales de l'économie publique pour les médecins indépendants}

Il est difficile de saisir les dépenses globales pour les médecins indépendants de Suisse, car la saisie de différents facteurs de calcul comme les frais du cabinet médical est liée à une grande imprécision.

Pour 2001 et 2002, les dépenses globales de l'économie publique pour les médecins indépendants s'élèvent respectivement à 10,016 et à 10,615 milliards de francs, soit $2,37 \%$ et $2,46 \%$ $\mathrm{du}$ produit intérieur brut de la Suisse.

Si nous prenons les chiffres d'Interpharma, en 2001 les 10,016 milliards correspondent à $21,71 \%$ et en 2002 , les 10,615 milliards à 22,12\% des coûts globaux de la santé.

Conformément à Pharma Information ${ }^{16}$ ), en 2001 les hôpitaux ont participé à hauteur de 24,236 milliards ou 52,54\% aux coûts globaux de la santé pour les traitements hospitaliers et ambulatoires. 
Selon la même source, en 2001 les dépenses globales pour les médecins indépendants ( $\mathrm{y}$ inclus la propharmacie) se sont élevés à 7,972 milliards ou 17,28\% des coûts globaux de la santé.

Le total des revenus de l'ensemble des médecins indépendants s'élève ainsi à 3,008 milliards ou $6,52 \%$ des coûts globaux de la santé.

Selon Pharma Information ${ }^{16)}$ à $^{22)}$, en 2002 les hôpitaux ont participé à hauteur de 25,644 milliards ou 53,45\% aux coûts globaux de la santé pour les traitements hospitaliers et ambulatoires.

Selon la même source, en 2002 les dépenses globales pour les médecins indépendants (y compris la propharmacie) se sont élevés à 8,102 milliards ou $16,89 \%$ des coûts globaux de la santé.

Le total des revenus de l'ensemble des médecins indépendants s'élève ainsi à 3,003 milliards ou $6,26 \%$ des coûts globaux de la santé.

\section{F.9. Tendances}

Durant la période 2001 à 2002, les revenus des médecins ont évolué pratiquement au même rythme que la hausse de l'indice du coût de la vie. Aucune augmentation supplémentaire de leurs revenus n'est à constater.

De manière générale, on peut constater que les revenus des médecins réalisant des actes techniques ont augmenté ces dernières années une nouvelle fois plus rapidement que ceux des médecins de premier recours.

Ces deux à quatre prochaines années, il sera intéressant d'évaluer les répercussions du TARMED, qui a aussi pour objectif d'abaisser la valeur des prestations médicales techniques au profit des prestations médicales intellectuelles.

L'imposition sur la base du revenu actuel permet donc d'analyser les revenus des médecins indépendants de Suisse chaque année et non plus seulement tous les deux ans.

\begin{tabular}{|c|c|c|c|c|c|c|c|}
\hline Canton & Nombre & 2001 & Indice & $+/-$ & Nombre & 2002 & Indice \\
\hline $\mathrm{ZH}$ & 2396 & 225092 & $106,8 \%$ & $-0,8 \%$ & 2227 & 223248 & $103,8 \%$ \\
\hline BE & 1727 & 204008 & $96,8 \%$ & $+8,4 \%$ & 1550 & 221106 & $102,8 \%$ \\
\hline LU & 415 & 229073 & $108,7 \%$ & $+2,1 \%$ & 384 & 233867 & $108,7 \%$ \\
\hline UR & 26 & 203565 & $96,6 \%$ & $-8,1 \%$ & 26 & 187142 & $87,0 \%$ \\
\hline SZ & 83 & 257363 & $122,1 \%$ & $-12,0 \%$ & 75 & 226608 & $105,4 \%$ \\
\hline UW & 74 & 210765 & $100,0 \%$ & $+1,6 \%$ & 68 & 214224 & $99,6 \%$ \\
\hline $\mathrm{GL}$ & 40 & 304498 & $144,5 \%$ & $-8,0 \%$ & 39 & 280213 & $130,3 \%$ \\
\hline ZG & 138 & 229936 & $109,1 \%$ & $+2,5 \%$ & 141 & 235740 & $109,6 \%$ \\
\hline FR & 321 & 206695 & $98,1 \%$ & $+10,2 \%$ & 319 & 227791 & $105,9 \%$ \\
\hline SO & 355 & 210676 & $100,0 \%$ & $+1,1 \%$ & 350 & 212984 & $99,0 \%$ \\
\hline BS & 446 & 170012 & $80,7 \%$ & $+3,7 \%$ & 386 & 176348 & $82,0 \%$ \\
\hline$B L$ & 471 & 236004 & $112,0 \%$ & $+2,6 \%$ & 474 & 242112 & $112,6 \%$ \\
\hline SH & 123 & 198933 & $94,4 \%$ & $+0,3 \%$ & 124 & 199583 & $92,8 \%$ \\
\hline AP & 97 & 212124 & $100,7 \%$ & $-0,9 \%$ & 88 & 210319 & $97,8 \%$ \\
\hline SG & 502 & 237025 & $112,5 \%$ & $-6,5 \%$ & 480 & 221620 & $103,0 \%$ \\
\hline GR & 275 & 205088 & $97,3 \%$ & $-2,5 \%$ & 243 & 199872 & $92,9 \%$ \\
\hline$A G$ & 598 & 202233 & $96,0 \%$ & $+0,6 \%$ & 482 & 203455 & $94,6 \%$ \\
\hline TG & 276 & 238948 & $113,4 \%$ & $-2,1 \%$ & 277 & 233993 & $108,8 \%$ \\
\hline TI & 372 & 241260 & $114,5 \%$ & $+4,3 \%$ & 369 & 251712 & $117,0 \%$ \\
\hline VD & 946 & 195247 & $92,7 \%$ & $+9,1 \%$ & 551 & 212952 & $99,0 \%$ \\
\hline VS & 359 & 161247 & $76,5 \%$ & $+9,4 \%$ & 323 & 176448 & $82,0 \%$ \\
\hline NE & 164 & 143656 & $68,2 \%$ & $+0,5 \%$ & 155 & 144313 & $67,1 \%$ \\
\hline GE & 183 & 163734 & $77,7 \%$ & $+10,6 \%$ & 150 & 181056 & $84,2 \%$ \\
\hline JU & 78 & 141574 & $67,2 \%$ & $+4,6 \%$ & 75 & 148044 & $68,8 \%$ \\
\hline Suisse & 10465 & 210723 & $100,0 \%$ & $+2,1 \%$ & 9356 & 215071 & $100,0 \%$ \\
\hline
\end{tabular}


Tableau 9

Revenu assujetti à I'AVS de tous les médecins indépendants âgés de moins de 66 ans, moyenne 2001 et 2002 d'après les spécialités.

\begin{tabular}{|c|c|c|c|c|c|c|c|}
\hline Spécialités & Nombre & 2001 & Indice & $+/-$ & Nombre & 2002 & Indice \\
\hline Anesthésie & 280 & 276861 & $131,4 \%$ & $-2,6 \%$ & 237 & 269546 & $125,3 \%$ \\
\hline Chirurgie & 505 & 259991 & $123,4 \%$ & $+5,5 \%$ & 445 & 274193 & $127,5 \%$ \\
\hline Dermatologie & 202 & 237186 & $112,6 \%$ & $+10,1 \%$ & 178 & 261041 & $121,4 \%$ \\
\hline Gynécologie & 648 & 249477 & $118,4 \%$ & $+1,3 \%$ & 590 & 252635 & $117,5 \%$ \\
\hline Médecine interne & 2054 & 217715 & $103,3 \%$ & $+1,6 \%$ & 1802 & 221116 & $102,8 \%$ \\
\hline Neurochirurgie & 22 & 309655 & $146,9 \%$ & $+18,3 \%$ & 23 & 366239 & $170,3 \%$ \\
\hline Neurologie & 134 & 185772 & $88,2 \%$ & $+3,1 \%$ & 120 & 191517 & $89,0 \%$ \\
\hline Ophtalmologie & 365 & 358039 & $169,9 \%$ & $+4,8 \%$ & 334 & 375067 & $174,4 \%$ \\
\hline Orthopédie & 247 & 284089 & $134,8 \%$ & $+6,4 \%$ & 230 & 302140 & $140,5 \%$ \\
\hline ORL & 187 & 286283 & $135,9 \%$ & $+6,4 \%$ & 166 & 304519 & $141,6 \%$ \\
\hline Pédiatrie & 511 & 170257 & $80,8 \%$ & $-1,7 \%$ & 464 & 167414 & $77,8 \%$ \\
\hline Médecine physique & 156 & 214994 & $102,0 \%$ & $+3,0 \%$ & 139 & 221448 & $103,0 \%$ \\
\hline Psychiatrie & 1265 & 117513 & $55,8 \%$ & $+1,0 \%$ & 1125 & 118679 & $55,2 \%$ \\
\hline Psychiatrie pour enfants & 192 & 102134 & $48,5 \%$ & $+1,9 \%$ & 173 & 104089 & $48,4 \%$ \\
\hline Radiologie & 127 & 391257 & $185,7 \%$ & $+8,6 \%$ & 111 & 424752 & $197,5 \%$ \\
\hline Urologie & 98 & 312410 & $148,3 \%$ & $+7,8 \%$ & 86 & 336784 & $156,6 \%$ \\
\hline Médecine centrale & 3460 & 194738 & $92,4 \%$ & $+0,0 \%$ & 3122 & 194787 & $90,6 \%$ \\
\hline Spécialistes & 7005 & 218618 & $103,7 \%$ & $+3,0 \%$ & 6234 & 225230 & $104,7 \%$ \\
\hline Médecine centrale & 3460 & 194738 & $92,4 \%$ & $+0,0 \%$ & 3122 & 194787 & $90,6 \%$ \\
\hline Tous & 10465 & 210723 & $100,0 \%$ & $+2,1 \%$ & 9356 & 215071 & $100,0 \%$ \\
\hline
\end{tabular}

\section{Illustration 13}

Evolution de la valeur moyenne du revenu assujetti à l'AVS des médecins indépendants de Suisse âgés de moins de 66 ans (nominal) 1971/72 à 2002.

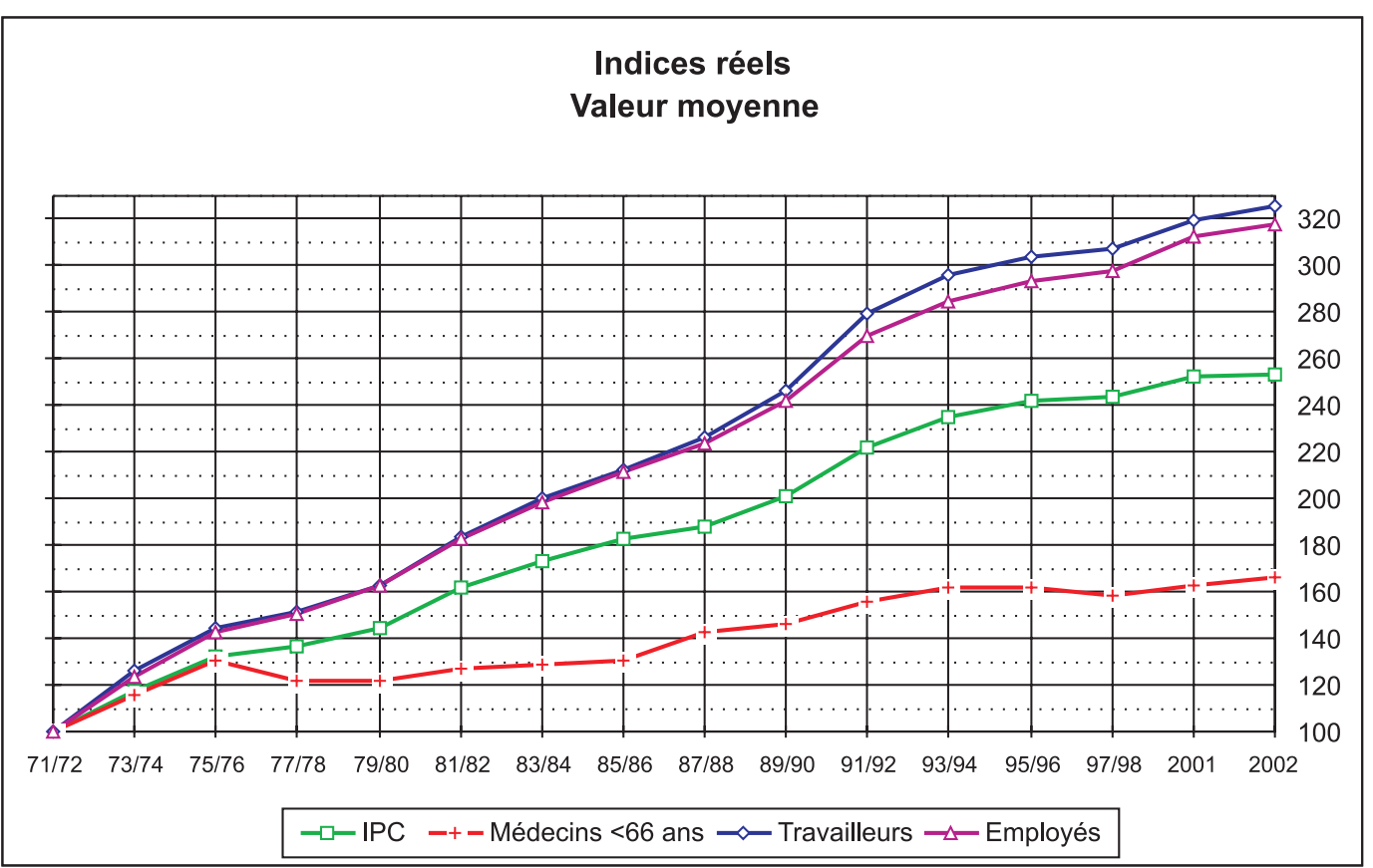


Illustration 14

Evolution de la valeur moyenne du revenu assujetti à l'AVS des médecins indépendants de Suisse âgés de moins de 66 ans (réel) 1971/72 à 2002.

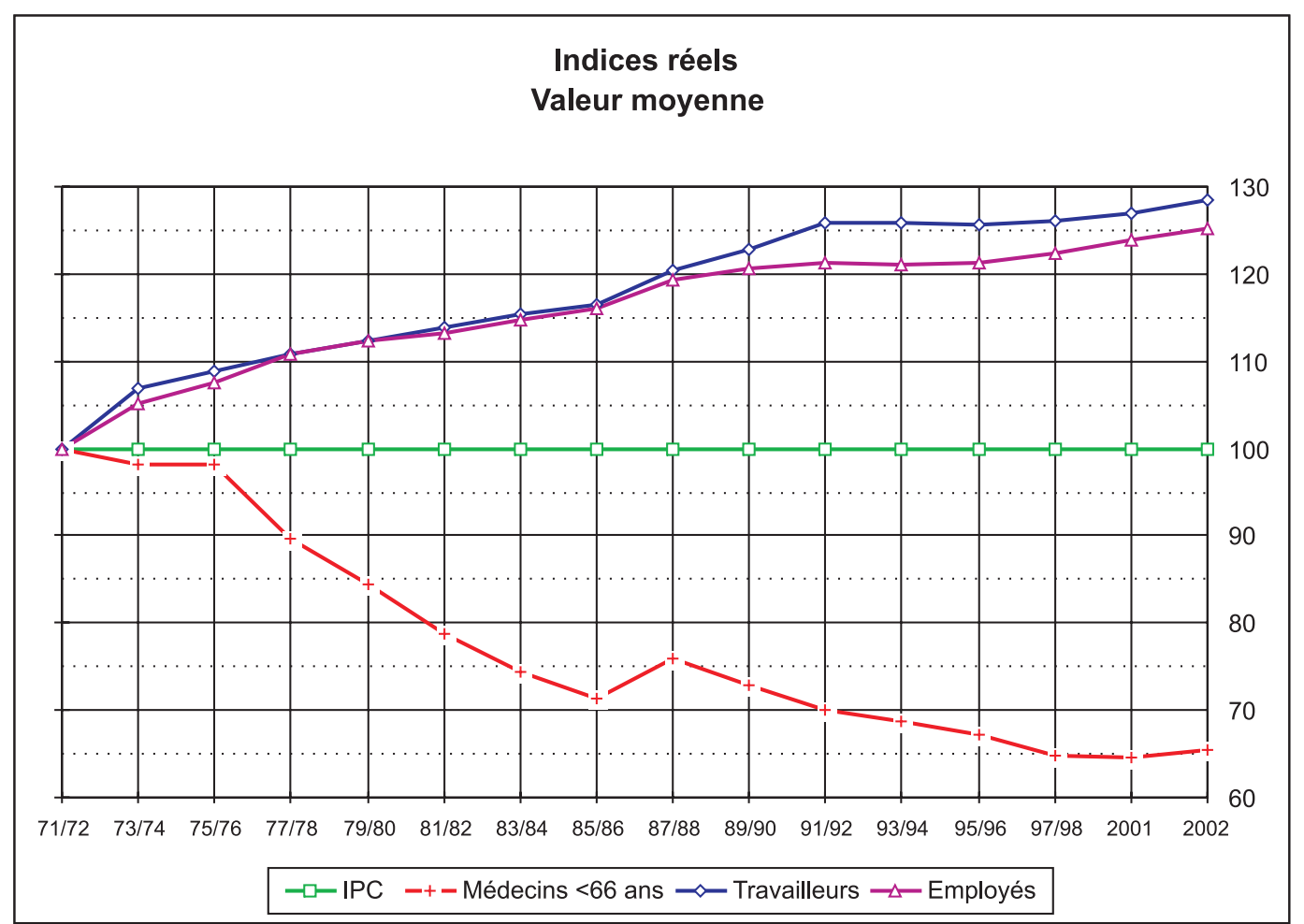

